\title{
Study of fusion regimes in an inertial electrostatic confinement device using the new eclipse disk diagnostic
}

\author{
S. Krupakar Murali, ${ }^{\text {a) }}$ B. B. Cipiti, ${ }^{\text {b) }}$ J. F. Santarius, and G. L. Kulcinski \\ Fusion Technology Institute, University of Wisconsin, 1500 Engineering Drive, Madison, Wisconsin 53706
}

(Received 13 January 2006; accepted 20 March 2006; published online 19 May 2006)

\begin{abstract}
New diagnostics are required to understand the physics operation of an inertial electrostatic confinement (IEC) device. In an attempt to understand the fusion source regimes within the IEC device, a new diagnostic called the eclipse disk has been introduced. This diagnostic was used to exploit the byproduct protons' energy difference between the deuterium-deuterium (D-D) and deuterium - an isotope of helium with two protons and one neutron (D- ${ }^{3} \mathrm{He}$ ) reactions to study the contributions of the protons generated from various source regimes. These source regimes are divided into five categories namely: converged core, embedded, beam background, volume, and wall-surface sources. The eclipse disk diagnostic has provided the first confirmed evidence that D- ${ }^{3} \mathrm{He}$ reactions are predominantly embedded reactions. It has been observed that at the present operating power levels $(6-10 \mathrm{~kW})$ most of the D-D reactions occur in the volume of the chamber caused by the charge exchanged neutrals, and the converged core contribution is significant only for D-D reactions. Since the branching ratio for the proton and neutron generation in a D-D fusion reaction is $\sim 50 \%$, it is inferred that the proton to neutron count ratio is a better parameter to monitor than either proton or neutron counts measured alone while studying the source regimes. This parameter may also be used for studying the potential wells within the cathode grid. (C) 2006 American Institute of Physics. [DOI: 10.1063/1.2200289]
\end{abstract}

\section{INTRODUCTION}

Unlike magnetic confinement and inertial confinement (laser, ions) fusion concepts, inertial electrostatic confinement (IEC) relies on the electrostatic field acceleration of ions to high energies for the generation of fusion. ${ }^{1-4}$ Though this device has been known for over four decades, little was done after 1975 and until the early 1990s. ${ }^{5-7}$ Several different versions of this concept are currently under investigation at various research institutes around the world. ${ }^{8-10}$ This device has been investigated using several different diagnostics, ${ }^{11,12}$ but is not yet completely understood. Due to the renewed interest in the near term applications of this device, ${ }^{13}$ a continual effort is being made to improve both the diagnostics and the experimental methods to understand this device. In the present paper we introduce a new diagnostic called the eclipse disk specifically to study the various modes of fusion within an IEC device. This diagnostic, as the name suggests, operates by eclipsing portions of the fusion reaction regime, allowing us to study the uneclipsed region independently.

Ions produced through electron impact ionization of the ambient gas inside the IEC chamber are subsequently accelerated toward the negatively charged central grid and gain fusion relevant energies. There are several modes by which fusion is known to occur in an IEC device (as illustrated in Fig. 1), namely, the following:

\footnotetext{
a) Telephone: (208) 850-2173. Electronic mail: ksubramanian@micron.com ${ }^{b)}$ Also at Sandia National Laboratory, P.O. Box 5800, MS 0748, Albuquerque, New Mexico 87185-0748. Telephone: (505) 284-8757. Electronic mail: bbcipit@sandia.gov
}

(1) Converged core (beam-beam). ${ }^{10}$

(2) Embedded (beam-target). ${ }^{10}$

(3) Volume source (charge-exchange-neutral). ${ }^{10}$

(4) Beam-background reactions that occur near and inside the cathode.

(5) Wall-surface reactions (charge-exchange-target).

In the converged-core mode, the ions converge inside the central grid and form a dense core. The streaming ions interact and cause fusion, primarily in the central region of the cathode. This is the most preferable mode of fusion production as the beam target is plasma and not the grid. The life of the system can be prolonged and the system efficiency would be optimized, if most of the reactions occurred in this mode.

The embedded source reactions occur between accelerated ions and those trapped in the wire. These reactions occur on the outer surface layers of the grid wire because the range of the $100 \mathrm{kV}{ }^{3} \mathrm{He}^{+}$ions in the $\mathrm{W}-25 \% \mathrm{Re}$ is only $\sim 0.25 \mu \mathrm{m}$. This mode is least preferable, as this mode leads to the erosion of the cathode grid and reduces the life of the system.

The volume source reactions occur everywhere inside the IEC device. In this mode the energetic ions chargeexchange with the background gas and the resulting fast neutrals undergo fusion with the neutral gas inside the chamber. These reactions are also preferable, but they reduce the efficiency of the device as the ions lose their energy to the charge exchanged neutrals even before falling through the full potential.

The beam-background reactions occur principally between the recirculating ions near the central grid and the background gas. Because a Child-Langmuir like electrostatic 


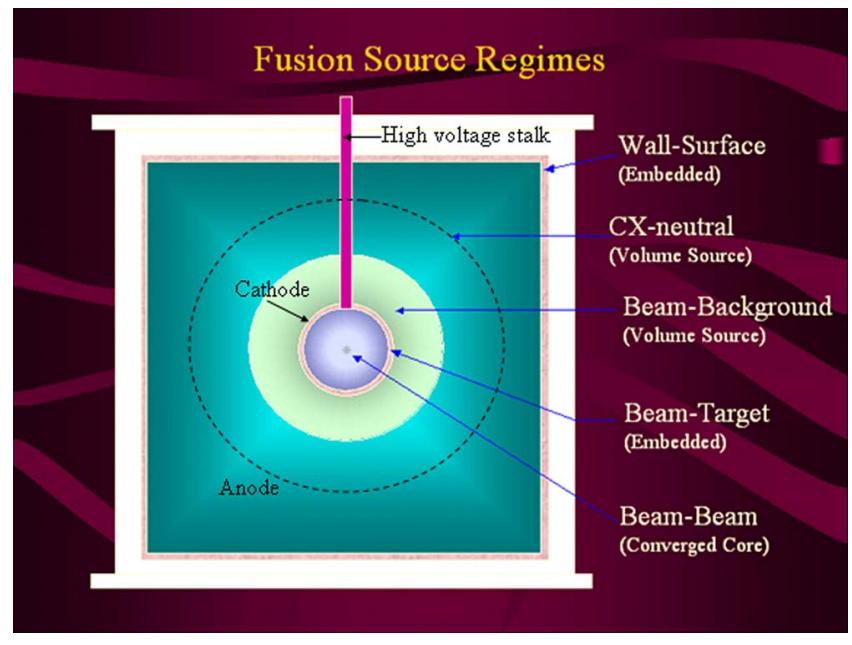

FIG. 1. Various fusion source regions in an IEC device.

potential prevails in the plasma, the ions gain most of their energy close to the central grid and as these ions recirculate they run into background gas and cause fusion. These reactions are more preferable than the volume source, but less preferable than the converged core source as the ions still lose energy through collisions with the neutral gas.

The wall-surface reactions occur between the atoms trapped in the surface layers of the chamber walls and the charge-exchanged neutrals. Although the wall-surface reactions appear to be not so significant (at the present power levels), it is speculated that at higher power and longer run times, the ${ }^{3} \mathrm{He}$ concentration trapped in the surface layers of the chamber walls would increase, especially if the chamber is made of stainless steel, and could potentially form stationary targets to the charge exchanged neutral deuterium atoms streaming outward and thereby cause fusion to occur on the wall surface. These reactions are preferable when compared to embedded reactions as these reactions erode the chamber walls and not the cathode, through sputtering.

Depending on the fuel, chamber pressure, power level of operation, and the kind of ionization source used, each of these source modes becomes important. For instance, at low pressures, the converged core and the embedded reactions dominate, but at higher pressures, the volume source dominates. Various fusion reactions (first and second generation fuels) shown in Table I for comparison. The difference in the energy of the protons released from the $\mathrm{D}-{ }^{3} \mathrm{He}$ and the parasitic D-D reactions that occur alongside $\mathrm{D}-{ }^{3} \mathrm{He}$ reactions (shown in Table I) are used to understand the various source regimes and are reported in the subsequent sections.

TABLE I. Key fusion reactions.

\footnotetext{
First generation fuels:

$\mathrm{D}+\mathrm{T} \rightarrow n(14.07 \mathrm{MeV})+{ }^{4} \mathrm{He}(3.52 \mathrm{MeV})$

$\mathrm{D}+\mathrm{D} \rightarrow n(2.45 \mathrm{MeV})+{ }^{3} \mathrm{He}(0.82 \mathrm{MeV})\{50 \%\}$$$
\rightarrow p(3.02 \mathrm{MeV})+T(1.01 \mathrm{MeV})[50 \%]
$$

Second generation fuel:

$\mathrm{D}+{ }^{3} \mathrm{He} \rightarrow p(14.68 \mathrm{MeV})+{ }^{4} \mathrm{He}(3.67 \mathrm{MeV})$
}

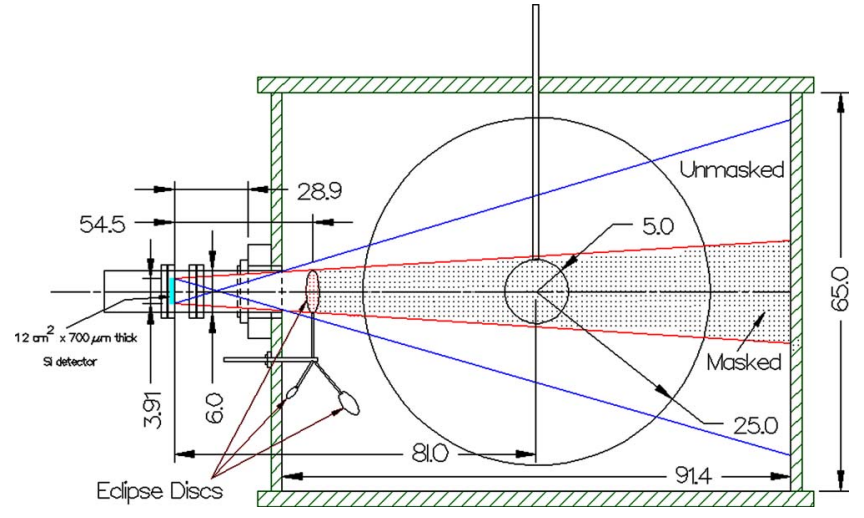

FIG. 2. Schematic of an eclipse disk diagnostic mounted on a port of the IEC chamber. Figure not to scale.

\section{ECLIPSE DISk DIAGNOSTIC}

There are two ways by which the fusion regimes can be studied: In the first method, the diagnostic measuring the fusion rate (e.g., a Si detector) focuses on a particular region of interest, while the second method involves blocking off the region of interest and measuring the fusion rate from the unblocked region. The difference between the fusion rates measured with and without blocking the region of interest gives the fusion rate from that region. Since it is much easier to block the region of interest, a new diagnostic known as Eclipse disk was developed. ${ }^{14}$ This diagnostic is so named because the disks manufactured using aluminum foil ( $0.5 \mathrm{~mm}$ thick) eclipse the region of interest in front of the proton detector (the region between the eclipse disk and the far wall).

Three different disks-large $(4.0 \mathrm{~cm}$ diameter), intermediate $(2.4 \mathrm{~cm})$, and small $(1.1 \mathrm{~cm})$ were used for the experiment shown schematically in Fig. 2. For the volumes blocked by each of these disks and the two dimensional 2D area projected at the center of the cathode calculations see Ref. 15. These eclipse disks can be positioned before the proton detector by rotating a lever from outside the chamber. The eclipse disk masks all the D-D fusion protons generated

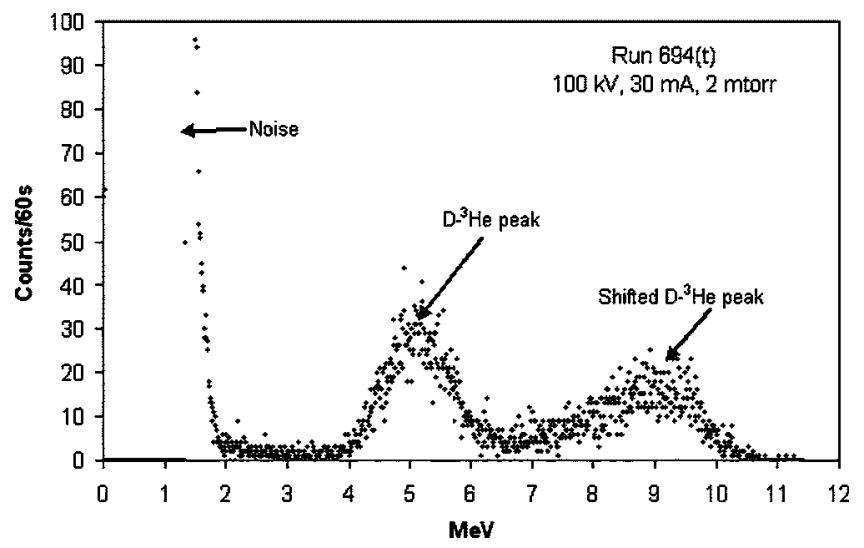

FIG. 3. A double peak is observed when $\mathrm{D}-{ }^{3} \mathrm{He}$ fuel is used in conjunction with an intermediate eclipse disk. The energy referred to in the Si detector measurements is the energy deposited into the detector, not the actual particle energy. 


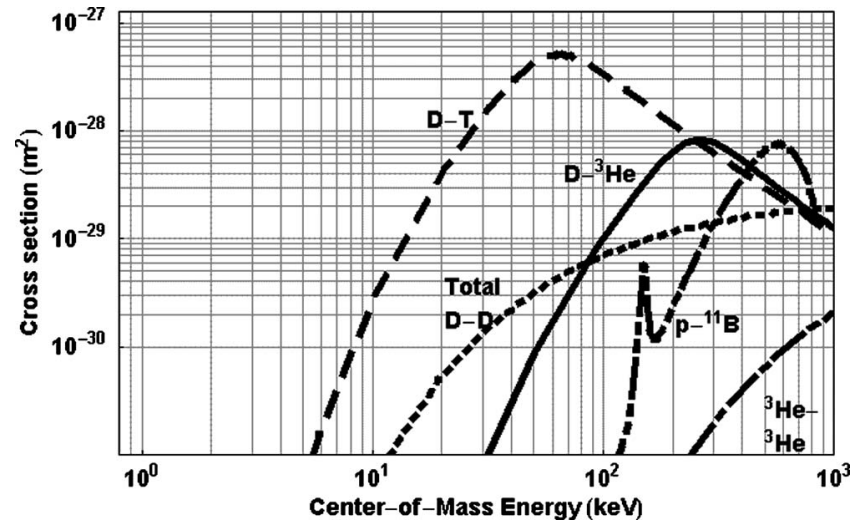

FIG. 4. As the initial energy of $\mathrm{D}-{ }^{3} \mathrm{He}$ protons decreases, it deposits more energy into the $700 \mu \mathrm{m}$ thick Si detector up to a maximum of $10 \mathrm{MeV}$ (Bragg peak), beyond which the proton deposits lesser energy.

between itself and the far wall; it is this volume that forms the region of interest. It is important to note that no fusion reactions occur in the region between the eclipse disk and the proton detector since this disk stops all the ions and the charge exchanged neutrals from entering this region. Also, the concentration of the embedded ${ }^{3} \mathrm{He}$ in the eclipse disk is very minimal. This is because the recirculation ${ }^{3} \mathrm{He}$ ion current is very small, and most of these ions get embedded on the cathode surface.

A disadvantage associated with this technique is that the eclipse disks are not highly selective; in other words they block more than the regions of interest. For instance, if the large disk is used in front of the proton detector to block the central grid, it not only blocks the contributions of the embedded fusion from the wires and the converged core, but also the volume source behind and in front of the central grid that falls in the masked region; see Fig. 1. Nevertheless, this technique can still be used to study the fusion regimes, as explained later.

Another interesting aspect of these disks (at the chosen thickness of $0.5 \mathrm{~mm}$ ) is that they do not completely block the energetic $\mathrm{D}-{ }^{3} \mathrm{He}$ protons, but only attenuate their energies. These attenuated protons show up as a different (shifted) energy peak in the proton energy spectrum, such as shown in Fig. 3. This is an important consequence of the eclipse disk because an independent confirmation of the protons coming from a region of interest is obtained from this shifted peak.

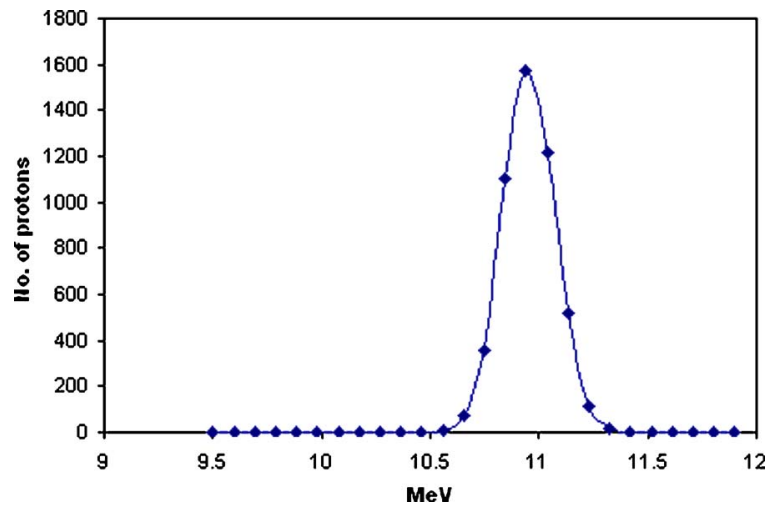

FIG. 6. Energy distribution of the $14.7 \mathrm{MeV}$ protons (exiting the $0.5 \mathrm{~mm}$ thick Al disk) calculated using SRIM. Also see Ref. 15.

The eclipse disk blocks most of the D-D protons (a few make it through the unmasked region), but it allows through all the $\mathrm{D}-{ }^{3} \mathrm{He}$ protons from both the unmasked and masked portions. However, those coming from the masked portion show up as a second peak in the proton energy spectrum; see Fig. 3. This is because the detector has a Bragg peak response, as shown in Fig. 4. As the D- ${ }^{3} \mathrm{He}$ protons lose energy, the amount of energy they deposit increases up to the Bragg peak value, which in the present case for the $700 \mu \mathrm{m}$ thick $\mathrm{Si}$ detector is $10 \mathrm{MeV}$, beyond which the energy deposited would once again begin to decrease.

Figure 5 shows the $\mathrm{D}-{ }^{3} \mathrm{He}$ proton scatter that causes the protons to ultimately deposit more energy into the detector. As the protons pass through the eclipse disk, the energy distribution on the exit end of the $\mathrm{Al}$ foil (calculated using the Monte Carlo SRIM code ${ }^{16}$ ) appears, as shown in Fig. 6.

As observed from Fig. 7, the protons have lower energy upon exiting the detector, and hence these protons would have deposited more energy (difference of energy peaks from Figs. 6 and 7) into the detector thus showing up at a higher energy level as the shifted peak in Fig. 3.

\section{RESULTS AND DISCUSSIONS}

Several experiments were performed using the eclipse disk diagnostic. In one set of experiments all three disks mentioned previously were used, as shown in Fig. 8 (where the view through the detector port with various disks is also
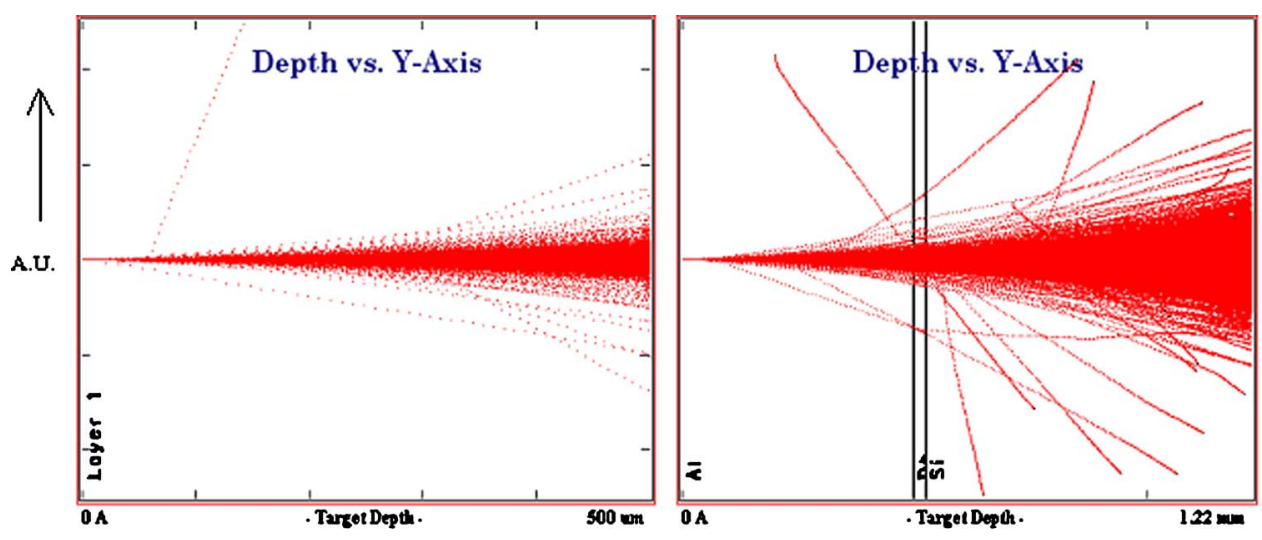

FIG. 5. Scattering of the D- ${ }^{3} \mathrm{He}$ protons as they pass through (a) $\mathrm{Al}$ $(0.5 \mathrm{~mm}$ thick) and (b) $\mathrm{Al}(0.5 \mathrm{~mm})$ $+\mathrm{Pb}(25 \mu \mathrm{m})+\mathrm{Si}$ detector $(700 \mu \mathrm{m})$; the $\mathrm{Al}$ disk is shown close to the $\mathrm{Pb}$ foil but is, in fact, $51 \mathrm{~cm}$ away from the $\mathrm{Pb}$ foil; hence some of the scattered protons could be lost before they reach the $\mathrm{Pb}$ foil. 


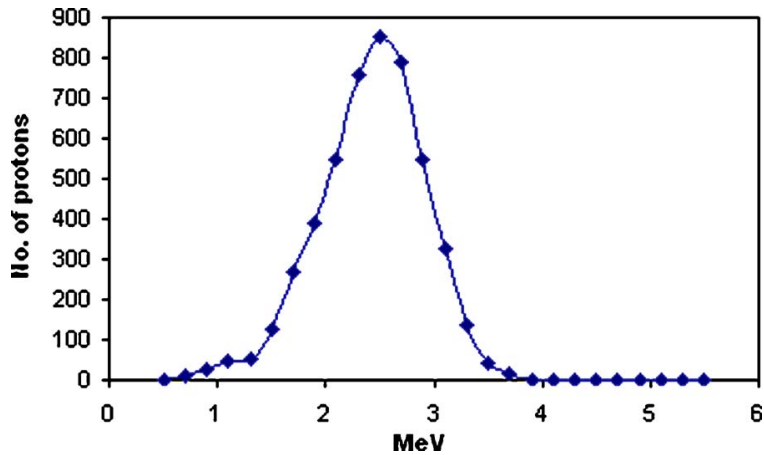

FIG. 7. Energy distribution of the protons exiting the Si detector in Fig. $5(\mathrm{~b})$.

illustrated). The results of these experiments performed with D- ${ }^{3} \mathrm{He}$ fuel mixture is shown in Fig. 9 (also see Ref. 17)

When $\mathrm{D}-{ }^{3} \mathrm{He}$ fuel is used in an IEC device, parasitic D-D reactions always occur alongside the $\mathrm{D}-{ }^{3} \mathrm{He}$ reactions. This aspect has been exploited in the present set of experiments to isolate and understand the contributions of the volume, embedded, and converged core regions. Figure 9 also shows the approximate volume eclipsed by each of the disks. The no eclipse case (as illustrated in Fig. 8) is normalized to $100 \%$ and all the other counts are relative to this value. When the large eclipse disk is used, this disk eclipses $19 \%$ of the volume visible to the proton detector (the total unmasked volume, Fig. 1), and as a result of this, more than $90 \%$ of the D-D protons are blocked. With the intermediate disk that eclipses $7.7 \%$ of the volume, $86 \%$ of the $\mathrm{D}-\mathrm{D}$ protons are blocked ( $14 \%$ shows up at the detector). With the small disk that eclipses just $0.26 \%$ of the volume, about $63 \%$ of the D-D protons are blocked (37\% shows up); this indicates that D-D reactions are predominant in the core region of the IEC device. The offset disk data that still eclipses $0.26 \%$ of the volume further supports this, as it was observed to block only $22 \%$ of the D-D reactions (78\% shows up). In order to understand the $\mathrm{D}-{ }^{3} \mathrm{He}$ reaction contributions a comparative study is performed later.

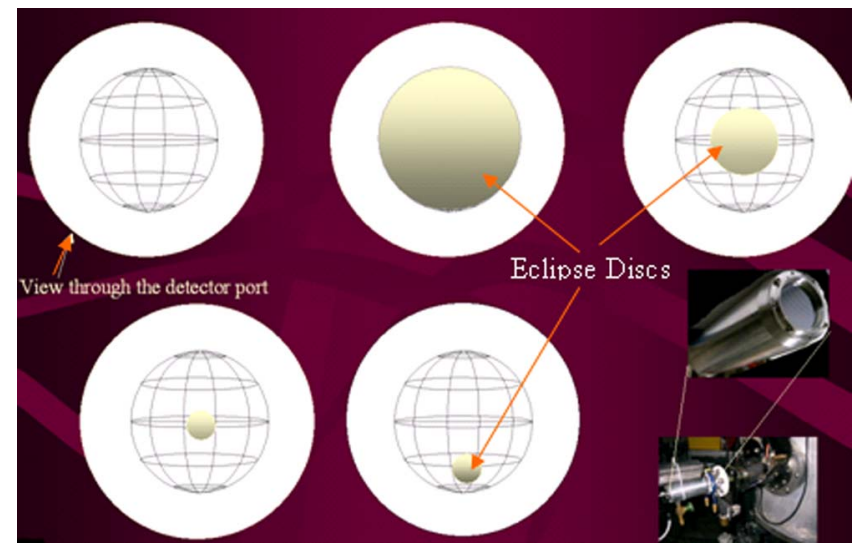

FIG. 8. (1) Unobstructed view through the detector port. (2) Large disk eclipsing the entire cathode. (3) Intermediate disk eclipsing the cathode. (4) Small disk eclipsing the core of the central grid. (5) Offset small disk eclipsing an arbitrary region of the cathode.

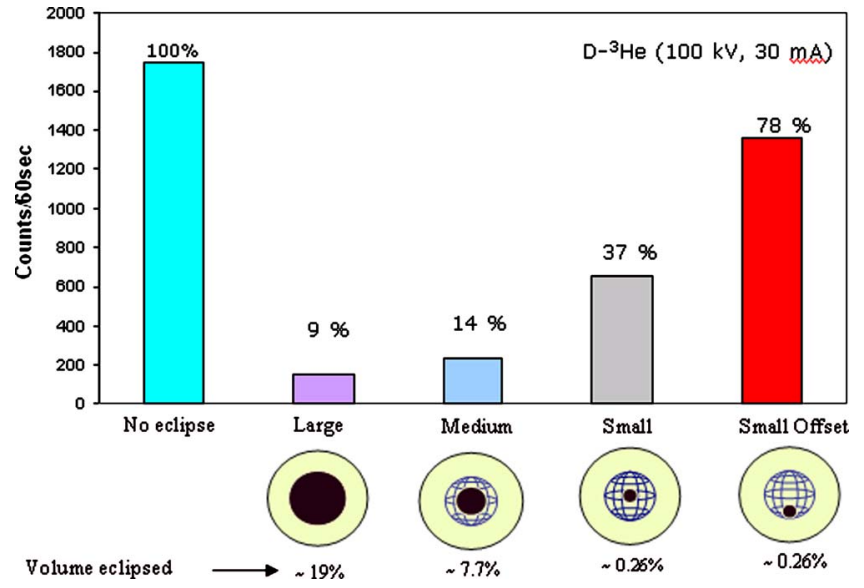

FIG. 9. Typical D-D proton count variation (integrating over all energies) with eclipse disks in an IEC device with $\mathrm{D}-{ }^{3} \mathrm{He}$ fuel at $100 \mathrm{kV}, 30 \mathrm{~mA}$. Also see Ref. 17.

The fusion occurring in each of the source regimes (tabulated below), contribute a certain percentage to the overall fusion rate detected by the Si detector. The 74\% D-D proton counts from the converged core means that this source regime contributes $74 \%$ of the total protons detected by the Si detector. The total contributions of each of the source regimes are tabulated in Table II.

As shown in Fig. 10 a comparison of the D-D reactions with $\mathrm{D}-{ }^{3} \mathrm{He}$ reactions shows several new features. The large disk blocks most of the D-D reactions and almost all of the $\mathrm{D}-{ }^{3} \mathrm{He}$ protons. The $\mathrm{D}-{ }^{3} \mathrm{He}$ protons that get through the eclipse disk shows up as a shifted peak, Fig. 3(a); some $\mathrm{D}-{ }^{3} \mathrm{He}$ protons are, however, lost, as they are scattered away by the eclipse disk. This suggests that most of the $\mathrm{D}-{ }^{3} \mathrm{He}$ reactions are contributions of either the converged core or the embedded reactions. Experiments with the small eclipse disk described below was used resolve the two contributions (converged core and the embedded source) for the $\mathrm{D}-{ }^{3} \mathrm{He}$ reactions.

The intermediate disk blocks $55 \%$ of the $\mathrm{D}-{ }^{3} \mathrm{He}$ protons and approximately $48 \%$ show up as the shifted proton peak. With the small eclipse disk, only $10 \%$ of the $\mathrm{D}-{ }^{3} \mathrm{He}$ protons are blocked, and even with the offset disk only $8 \%$ of the protons are blocked. Hence the D- ${ }^{3} \mathrm{He}$ reactions are dominated by the embedded reactions. This is the first confirmed evidence that $\mathrm{D}-{ }^{3} \mathrm{He}$ reactions are predominantly embedded reactions in a gridded IEC device.

Although the fraction of ${ }^{3} \mathrm{He}$ ions is small (due to the Penning ionization effect ${ }^{18}$ ) in the $\mathrm{D}-{ }^{3} \mathrm{He}$ mixture, those ions that survive quickly embed themselves on the surface of

TABLE II. Fusion source regime contributions to the overall proton rate detected by the Si detector. The error in these contributions is $\pm 2 \%$.

\begin{tabular}{ccc}
\hline \hline Fusion source regime & DD & D- ${ }^{3} \mathrm{He}$ \\
\hline Converged core & $20 \%-22 \%$ & $5 \%-7 \%$ \\
Embedded & $5 \%-8 \%$ & $93 \%-96 \%$ \\
Volume & $67 \%-70 \%$ & Negligible \\
Wall surface & Negligible & Negligible \\
\hline \hline
\end{tabular}




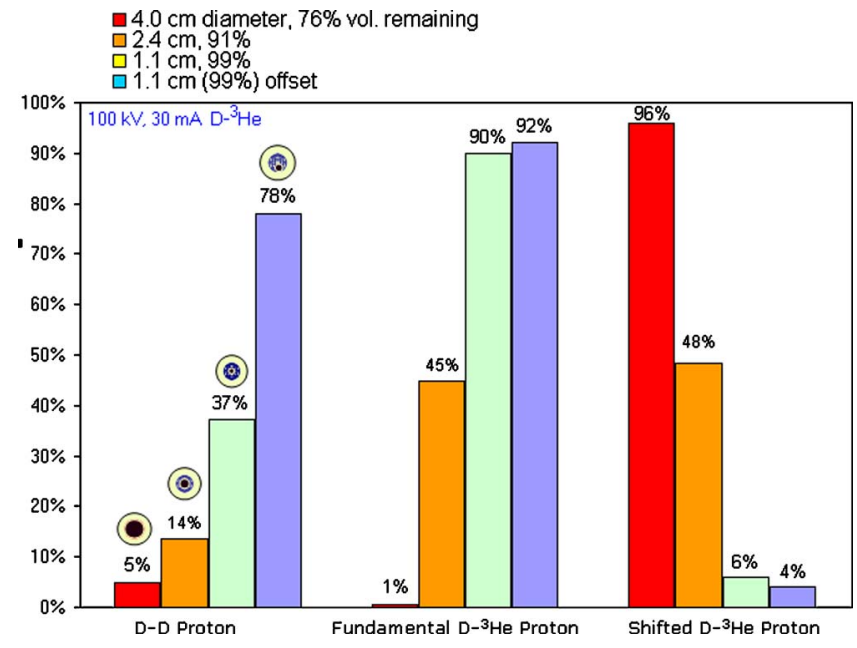

FIG. 10. The percent of protons detected by the detector corresponding to each of the eclipse disks.

the cathode grid wires forming stationary targets for the oncoming deuterium ions. Another independent experiment performed using solid targets ${ }^{19}$ provided further evidence that the $\mathrm{D}-{ }^{3} \mathrm{He}$ reactions are embedded (beam-target reactions) in nature.

\section{A. Eclipse disk scan experiment}

The set of experiments described in this section is an extension of the offset small eclipse disk experiment described earlier, wherein the small disk is scanned all the way across the central grid in small increments, as shown in Fig. 11. The area blocked by the disk in each position overlaps with the previous area, but there is no overlap between alternate disk positions, as shown in Fig. 12.

Proton and neutron data are recorded at each position of the disk; Fig. 13 shows the plot of protons/s vs position of the eclipse disk at various voltages, using D-D fuel. It is observed that the protons/s converge at position 5, showing that converged core contributions exist for D-D reactions. The magnitude of the proton rate (protons/s) increases with voltage because the ion energy (and hence the reaction crosssection) increases with voltage; see Fig. 14.

\section{B. Effects of voltage and pressure on the fusion convergence}

The proton rate shows the convergence (more protons produced in the core) as the disk scans the central grid. In-

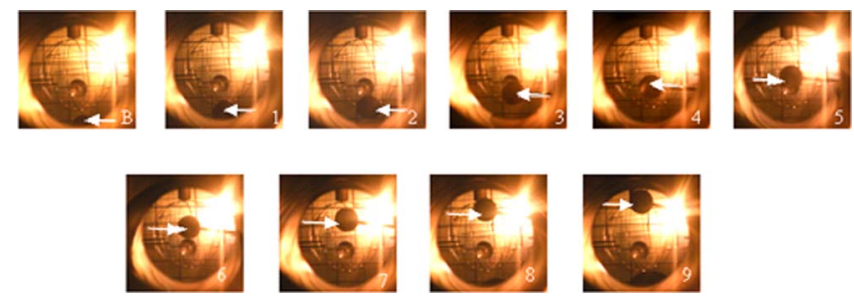

FIG. 11. Pictures taken through the detector port of the small disk at various locations. The bright light in the background comes from the filament electron source in the far end.
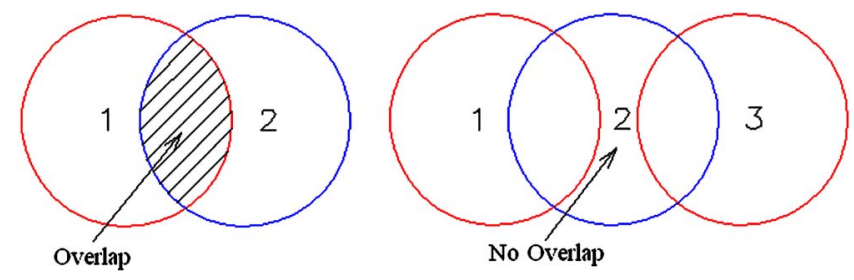

FIG. 12. There is $\sim 50 \%$ overlap between two consecutive disks [red (1) and blue (2)], but not between every other disk position [in red (1) and (3)].

terestingly, when the protons are normalized to 1 , the proton counts show a regular structure, and even more interesting is the fact that other than the counts at $40 \mathrm{kV}$, all the counts seem to show a dip at the position (2) in Fig. 15. If only half the grids were scanned (assuming symmetry), this dip could be easily mistaken to be a double well structure. Some careful studies ${ }^{20}$ of the double well structures in an IEC device have overlooked this aspect and may be a cause of concern. The statistical variation in the operational parameters could cause such anomalous structures in Fig. 15.

The neutron data is independent of the position of the disk and would increase or decrease with the total number of D-D protons because the branching ratio for the D-D reactions is very close to $50 \%$, as shown in Table I. Any changes in the neutron counts may be caused due to variations in any of the following: grid temperature, chamber pressure, drifts in grid voltage, and/or drifts in current. These factors would also affect the protons, but proton and neutron counts would follow each other for D-D reactions. Hence a new parameter (proton count to neutron count ratio) is proposed in the next section.

\section{Proton/neutron count $(\mathrm{P} / \mathrm{N})$ ratio}

If the total number of proton/s $(\mathrm{P})$ from the $\mathrm{D}-\mathrm{D}$ reactions were divided by the (simultaneously recorded) total number of neutrons $(\mathrm{N})$ from $\mathrm{D}-\mathrm{D}$ reactions $\left(\mathrm{D}-{ }^{3} \mathrm{He}\right.$ reactions do not produce neutrons, and the protons are of much higher energy; see Table I) at a given position of the disk, then the variation of this parameter $(\mathrm{P} / \mathrm{N}$ ratio) with the position of the disk would be a true indicator of any anomalous behavior of the protons generated in the central grid region.

The $\mathrm{P} / \mathrm{N}$ ratio assumes a constant calibration factor, i.e., constant contributions from each type of source with voltage

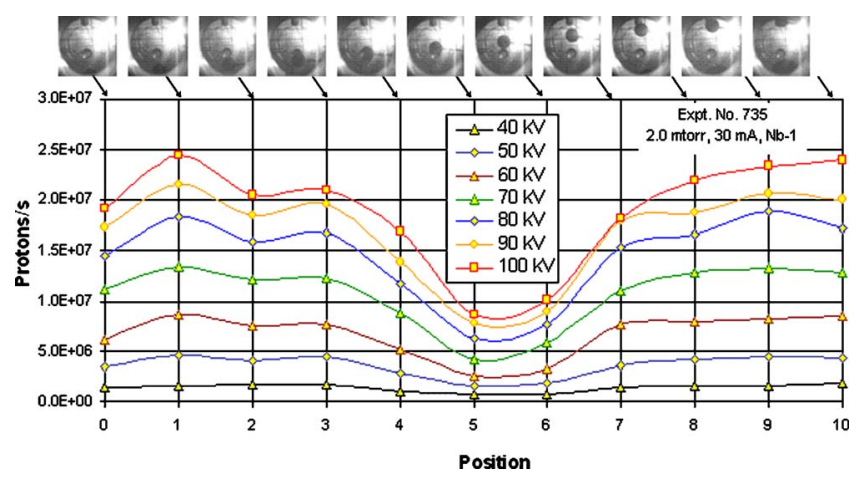

FIG. 13. D-D protons/s at various voltages versus position. 


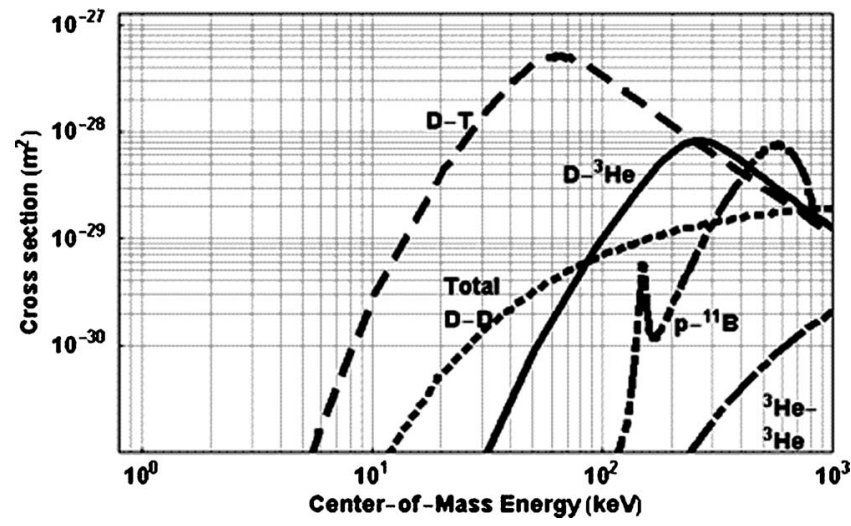

FIG. 14. Fusion reaction rate versus ion temperature.

and current. This is supported by the observation that the fusion rate observed from various regions using the eclipse disk changes linearly with voltage and there is no significant deviation from the relative proportions at various voltages up to $100 \mathrm{kV}$, beyond which experiments were not performed.

Figure 16 is a plot of the neutrons/s vs position. The counts are not constant during a given scan and show some statistical variations. These variations are present, although not apparent in the proton counts in Fig. 15. Hence, a P/N ratio would normalize the statistical variations during the experiment. Neutron counts tend to track the statistical variations just as do the protons, and hence a ratio of the two would normalize any statistical variations.

Although pressure normalized proton data was used for a proton collimation study in the literature, ${ }^{3}$ the $\mathrm{P} / \mathrm{N}$ ratio should be preferred because it accounts for all the parameters that vary during the experiment (not just the pressure).

Figure 17 shows the P/N ratios normalized to the maximum value in a particular scan (at a constant voltage) versus position at various voltages. Figure 17 shows that there is no significant improvement in convergence with voltage. The overall reaction rate increases but the relative number of reactions that occurs in the core of the device remains relatively constant. The dip that we had seen earlier at position 2 no longer exists in the $\mathrm{P} / \mathrm{N}$ ratio scan and is concluded to be an artifact of the experiment, created by the variation in the parameters such as chamber pressure, drifts in the cathode voltage, and drifts in the cathode current.

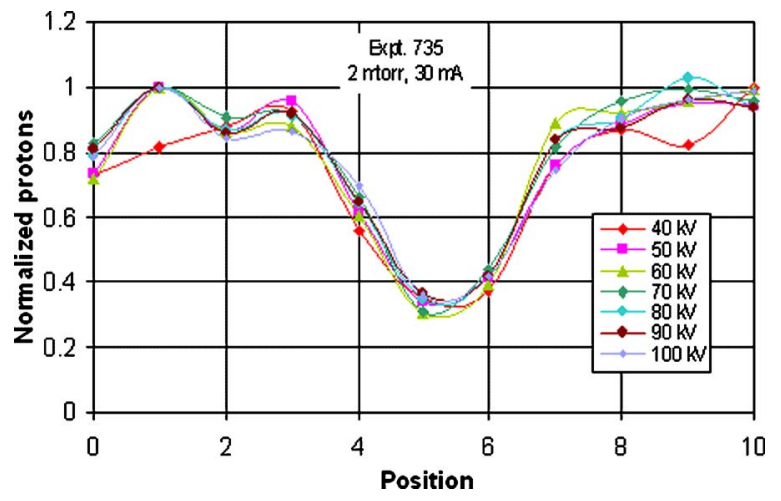

FIG. 15. Normalized D-D proton counts versus position at various voltages.

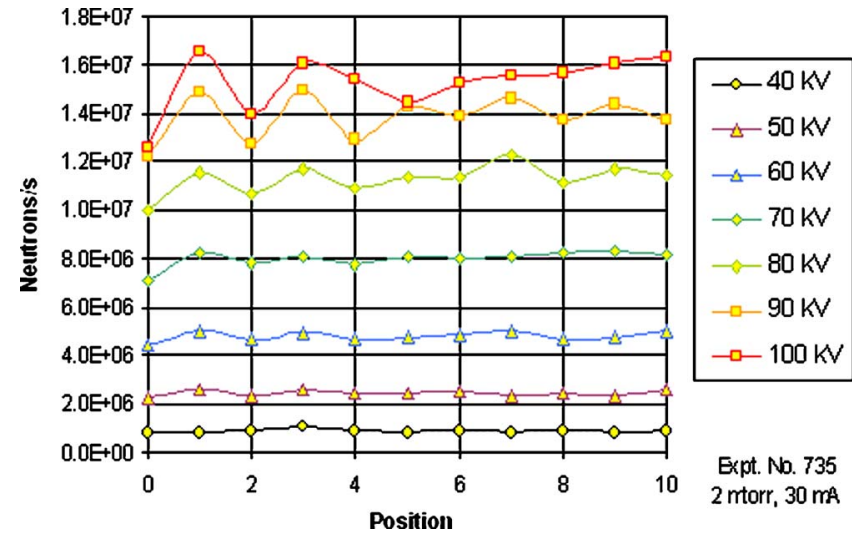

FIG. 16. D-D neutrons/s at various voltages versus position.

The error bars for Fig. 17 are generated by dividing the maximum proton count [(original)counts + sqrt (counts)] by minimum neutron counts [(original)counts-sqrt(counts)] at a particular voltage. Furthermore, there is negligible error in the $x$ direction because the eclipse positions are repeatable.

There is $\sim 50 \%$ overlap between consecutive disk positions, as illustrated in Fig. 2, and it is evident from Fig. 17 that the converged core boundary lies between positions 5 and 6, where the number of counts masked is the maximum. Since the counts do not return to the maximum value as in position 4 , the converged core is still eclipsed in position (7)

Approximating the volume eclipsed by the small disk to be a cylinder ${ }^{15}$ of diameter $1.1 \mathrm{~cm}$, the upper limit of the diameter of the converged core is the separation distance of the overlap regions shown in Fig. 12, it seems that the size of the converged core diameter is less than $0.55 \mathrm{~cm}$.

\section{Conclusions}

With the development of this new eclipse disk diagnostic, a new understanding of the source regimes within the IEC device has been made possible. Eclipse experiments (with $\mathrm{D}-{ }^{3} \mathrm{He}$ fuel) revealed that a converged core exists for the D-D reactions and not for $\mathrm{D}-{ }^{3} \mathrm{He}$ reactions (is negligible). Such a converged core is small $(<0.55 \mathrm{~cm})$ compared to the cathode grid diameter $(10 \mathrm{~cm})$. D- ${ }^{3} \mathrm{He}$ reactions are predominantly embedded reactions because the large disk masks out only $19 \%$ of the volume but shields out $\sim 90 \%$ of

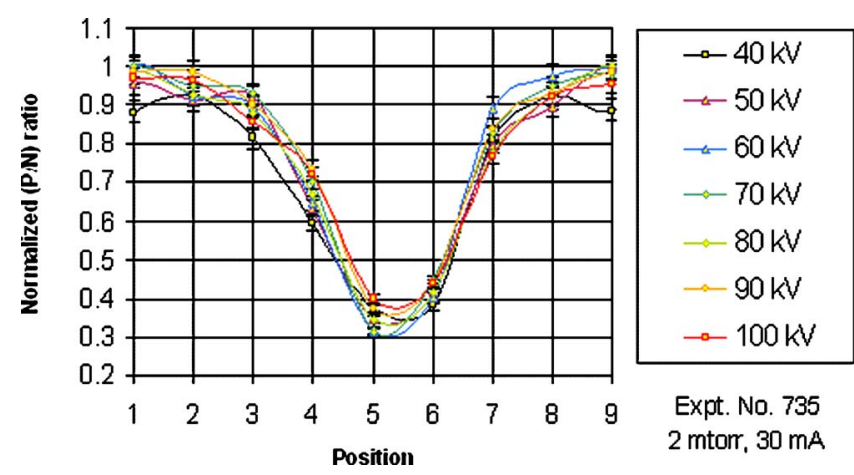

FIG. 17. Normalized (P/N) ratio versus position at various voltages. 
the fusion protons. The wall-surface contributions for both D-D and D- ${ }^{3} \mathrm{He}$ reactions are minimal at the present power levels.

While the D-D protons deposit all their energy (after losing some to the $\mathrm{Pb}$ foil) into the Si detector, the $\mathrm{D}-{ }^{3} \mathrm{He}$ protons deposit energy only partially. The proton to neutron ratio is a better parameter to monitor (especially when D-D fuel is used) while studying the fusion sources as the proton to neutron branching ratio is $50 \%$ for D-D fusion. This is especially true while attempting to measure potential wells using proton rate generation. Further research is required to resolve the contributions of the beam-background reactions from the rest of the source regimes.

\section{ACKNOWLEDGMENTS}

We would like to thank R. P. Ashley, Greg Pierfer, Ross Radel, John Weidner, Tracy Radel, Dave Boris, and Alex Wehmeyer for helping us run the experiments and useful discussions.

We thankfully acknowledge Grainger Foundation and Wilson Greatbatch for funding the present research.

${ }^{1}$ O. A. Lavrent'ev, Ukr. Fiz. Zh. 8, 440 (1963).

${ }^{2} \mathrm{P}$. T. Farnsworth, "Method and apparatus for producing nuclear-fusion reactions," U.S. Patent \#3,386,883, 1968.

${ }^{3}$ R. L. Hirsch, J. Appl. Phys. 38, 452 (1967).

${ }^{4}$ W. C. Elmore, J. L. Tuck, and K. M. Watson, Phys. Fluids 2, 239 (1959).
${ }^{5}$ J. H. Nadler, G. H. Miley, Y. B. Gu, and T. Hochberg, Fusion Technol. 20, 850 (1991)

${ }^{6}$ G. H. Miley, J. Nadler, T. Hochberg, Y. B. Gu, and O. Barnouin, Bull. Am. Phys. Soc. 35, 2138 (1990).

${ }^{7}$ T. A. Thorson, R. D. Durst, R. J. Fonck, and A. C. Sontag, Nucl. Fusion 38, 4 (1998).

${ }^{8}$ J. Park, R. A. Nebel, S. Stange, and S. K. Murali, Phys. Rev. Lett. 95, 015003 (2005).

${ }^{9}$ K. Yoshikawa, K. Masuda, H. Toku, K. Nagasaki, T. Mizutani, T. Takamatsu, M. Imoto, Y. Yamamoto, M. Ohnishi, H. Osawa, E. Hotta, T. Kohno, A. Okino, M. Watanabe, K. Yamauchi, M. Yuura, S. Shiroya, T. Misawa, and T. Mori, Fusion Sci. Technol. 47, 1224 (2005).

${ }^{10}$ J. F. Santarius, G. L. Kulcinski, R. P. Ashley, D. R. Boris, B. B. Cipiti, S. Krupakar Murali, G. R. Piefer, R. F. Radel, T. E. Radel, and A. L. Wehmeyer, Fusion Sci. Technol. 47, 1238 (2005).

${ }^{11}$ D. A. Swanson, B. E. Cherrington, and J. T. Verdeyen, Phys. Fluids 16, 1939 (1973).

${ }^{12}$ K. Yoshikawa, K. Takiyama, K. Masuda, H. Toku, T. Koyama, K. Taruya, H. Hashimoto, A. Nagafuchi, T. Mizutani, Y. Yamamoto, M. Ohnishi, H. Horiike, and N. Inoue, Fusion Technol. 39, 486 (2001).

${ }^{13}$ G. L. Kulcinski, J. Weidner, B. Cipiti, R. P. Ashley, J. F. Santarius, S. K. Murali, G. Piefer, and R. Radel, Fusion Sci. Technol. 44, 559 (2003).

${ }^{14}$ R. P. Ashley, G. L. Kulcinski, J. F. Santarius, S. Krupakar Murali, G. Piefer, B. B. Cipiti, and R. Radel, Fusion Sci. Technol. 44, 559 (2003).

${ }^{15} \mathrm{~S}$. Krupakar Murali, "Diagnostic study of advanced fuel fusion in an IEC device," Ph.D. dissertation, University of Wisconsin, Madison, 2004.

${ }^{16}$ J. F. Ziegler, "The stopping and range of ions in matter," Instruction manual, Ver. 96. xx, 25 February 2004.

${ }^{17}$ R. P. Ashley, G. L. Kulcinski, J. F. Santarius, S. Krupakar Murali, G. Piefer, B. B. Cipiti, and R. F. Radel, 5th US-Japan Workshop, Madison, WI, 2003.

${ }^{18}$ F. M. Penning, Physica (The Hague) 3, 873 (1936).

${ }^{19}$ B. B. Cipiti, Ph.D. thesis, University of Wisconsin, Madison, 2004.

${ }^{20}$ G. Yibin, Ph.D. thesis, University of Illinois at Urbana-Champaign, 2002. 